\title{
Secondary Data Analysis: Lessons and perspective of a research parasite
}

\author{
Ayush T Raman ${ }^{1,2}$ \\ 1 Broad Institute of Harvard and MIT, Cambridge, MA, USA \\ 2 Department of Pathology and Center for Cancer Research, Massachusetts General Hospital \\ and Harvard Medical School, Boston, MA, USA
}

Correspondence address: aayushraman09@gmail.com, https://orcid.org/0000-0003-2872-2307

\begin{abstract}
Secondary data analysis refers to re-analyzing publicly available datasets to investigate the questions that original scientists had not posited. This helps in scientific progress by paving the path to more reliable and robust analyses and new research directions without any considerable expense. However, these datasets are anything but perfect, and researchers must investigate and assess the signal to noise ratio robustly to extract meaningful information. These efforts of rigorous secondary analysis are further recognized and supported by the Research Parasite Awards. As the 2020 Junior Research Parasite Award recipient, I share my journey and perspective of a research parasite in this commentary article.
\end{abstract}

\section{Introduction}

My journey as a research parasite started with reading a paper that suggested genes longer than $100 \mathrm{~kb}$ are preferentially misregulated due to the loss of function mutations in the MECP2 gene [1]. It is a methyl-binding protein whose task is to orchestrate gene expression, and MeCP2 mutations disrupt the expression of several thousand genes. This was an intriguing observation that garnered much attention, but it was also biologically puzzling as to why very long genes would be more likely to be misregulated even if neuronal genes tend to be larger than genes in other bodily tissues [2]. This led me to quest after the mechanism behind this result and further investigate the function of MeCP2 in neuronal tissues.

I re-analyzed different MeCP2 datasets and used their approach to reproduce the plots and results from their paper. While pursuing this exercise, I realized that these previous studies did not use any statistical methods to establish a baseline (i.e., intra-sample variations) against which the significance of the length-dependent changes could be measured. When we established the baseline variation first, the apparent bias toward long genes disappeared. I then went a step further and examined benchmark transcriptomic datasets that did not include MeCP2 or its effects on expression at all -- and found that these benchmark datasets were also prone to long gene bias. This led us to hypothesize that the "long gene trend" might be an artifact of the microarray and RNA-seq technologies used to measure gene expression changes because they require PCR gene amplification. When we analyzed gene sets on the NanoString platform (which is not amplification-based), long genes were no more likely to be affected by MeCP2 mutations than genes of shorter length. This confirmed our notion that the previous observations of long-gene bias resulted from amplification-based technologies and the failure to establish a proper baseline [3]. I believe our secondary analysis will help the scientific 
community think about more reliable and robust analyses for amplification-based sequencing data.

As the 2020 Junior Research Parasite award recipient, I was recognized for my contribution to rigorous secondary analysis at the Pacific Symposium on Biocomputing (PSB) conference, 2020. Since 2017 this honor is awarded annually and is supported by the Gigascience and Gordon and Betty Moore Foundation [4, 5]. This commentary article will share my perspective and lessons learned while mining and re-analyzing the datasets.

\section{Understand the dataset and statistical methods used by original researchers}

As a data scientist, it is very tempting to download the dataset from public repositories such as Gene Expression Omnibus and apply the choice of algorithms one is interested in. However, a comprehensive understanding of the dataset is necessary [5] to generate hypotheses and questions that need to be answered. This includes defining the dataset in terms of outcome variables (ex: gene expression datasets), different biological (ex: genotype or phenotype information) and confounding covariates (ex: data generated using different sequencing platforms or across different days). Other pertinent details that research parasites should carefully review are statistical methods and the underlying assumptions associated with them that original researchers used, such as normality, heteroskedasticity and independence of observations. These pieces of information establish a strong foundation for further downstream analyses.

\section{Exploratory Data analysis helps deconvolute the assumptions}

Exploratory data analysis (EDA) using different visualization techniques should be the first step in understanding the characteristics and possible insights about the dataset. Box plots, histograms and scatter plots are essentially the first step to gain intuition about the dataset. For example, it helps understand if the dataset is normalized or not, what are the data distribution and the total number of features in an assay. Next, high dimensionality reduction methods, including Principal Component Analysis (PCA) and Multi-dimensionality scaling (MDS), are great tools to visualize the dataset. An adequately labeled PCA plot has the power to provide information such as -- a) presence of batch effects [6], b) labeling errors in the metadata sheet, c) inter and intra sample variations, and d) informative features that can distinguish the groups of samples (Fig 1A).

During this project, I wrote scripts that automate exploratory data analysis for all the datasets to investigate the plots methodically. Moreover, it also allowed my analysis to be consistent across different datasets of the same type. One of the "eureka moment" we obtained while performing such analysis was that the previous studies assumed intra-sample variation within the control group to be equal to zero. This realization helped us develop a statistical approach to accurately estimate intra-sample variation against which the significance of the length-dependent changes in mutant samples was measured [3]. Therefore, EDA help reveals the degree of variability among different sample groups and inform further analyses required to investigate the background noise.

\section{Use of benchmark datasets to determine the ground truth}

Since most scientific studies consist of small sample sizes or low statistical power [7], a well-characterized benchmark dataset would provide accurate and unbiased results and help generate new hypotheses. In my project, we took advantage of SEQC consortium datasets [8] 
to uncover that one of the major sources of technical variation is intra-sample variation and postulated that PCR amplification bias could amplify long genes analysis that was confirmed using NanoString platform, a non-amplification based assay.

\section{Encourage, support and fund research related to negative results}

Even though peer reviews evaluate and improve the quality of the manuscript, a recent survey of more than 1500 scientists has raised the concern about the problems with reproducibility in biomedical sciences [9]. This could be due to various reasons, including errors during data generation, lack of transparency in documenting methods, code and data availability, and personal biases [6]. One of the other yet subtle reason behind this crisis [9] is the absence of studies highlighting negative results in high impact journals, sometimes referred to as "publication bias". This is because the editors and peer-reviewers are biased towards publishing positive new results, possibly because they are cited more than a negative result [6]. However, there is a massive downside of the negative results not getting published. There is a possibility of entering into a vicious cycle of wrong scientific direction that may lead to failed experiments, waste of public funds, and delay in scientific progress [10].

I think the change can only be achieved if we advocate for open and reproducible science $[4,5]$. This can be achieved by documenting our methods, making our source code and dataset easily accessible to the community for effective and reproducible science. Moreover, we should be thankful and indebted to the researchers who generated and made the dataset accessible to the community, without which our research would not have been possible.

A

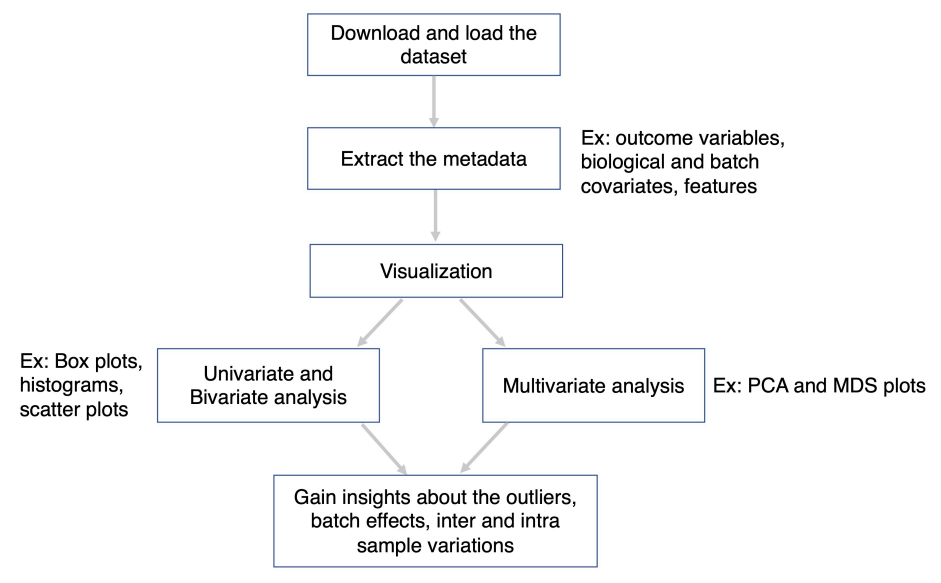

B

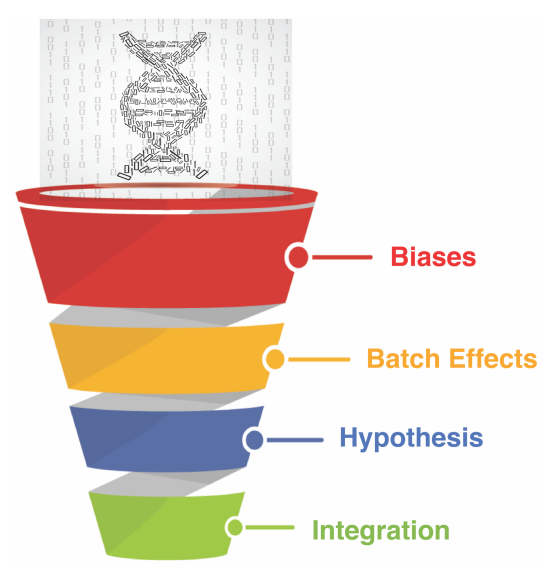

Figure 1: A) Schematic of a subset of steps involved in the workflow of the exploratory data analysis. Each box contains a description of the data analysis step and arrows indicate the progression through the analysis workflow. Ex means example of such data analysis step. B) Schematic workflow of a typical secondary analysis project where big data is first analyzed to detect biases, batch effects in the dataset, gain insights \& generate hypothesis and integrate different datasets to get optimal results. 


\section{References}

1. Gabel HW, Kinde B, Stroud H, Gilbert CS, Harmin DA, Kastan NR, Hemberg M, Ebert $\mathrm{DH}$, Greenberg ME: Disruption of DNA-methylation-dependent long gene repression in Rett syndrome. Nature 2015, 522(7554):89-93.

2. Zylka MJ, Simon JM, Philpot BD: Gene Length Matters in Neurons. Neuron 2015, 86:353-355.

3. Raman AT, Pohodich AE, Wan YW, Yalamanchili HK, Lowry WE, Zoghbi HY, Liu Z: Apparent bias toward long gene misregulation in MeCP2 syndromes disappears after controlling for baseline variations. Nat Commun 2018, 9(1):3225.

4. Greene CS, Garmire LX, Gilbert JA, Ritchie MD, Hunter LE: Celebrating parasites. Nat Genet 2017, 49(4):483-484.

5. Duvallet C: Data detectives, self-love, and humility: a research parasite's perspective. Gigascience 2020, 9(1).

6. Leek JT, Peng RD: Opinion: Reproducible research can still be wrong: adopting a prevention approach. Proc Natl Acad Sci 2015, 112(6):1645-1646.

7. Button KS, loannidis JP, Mokrysz C, Nosek BA, Flint J, Robinson ES, Munafo MR: Power failure: why small sample size undermines the reliability of neuroscience. Nat Rev Neurosci 2013, 14(5):365-376.

8. SEQC/MAQC-III C: A comprehensive assessment of RNA-seq accuracy, reproducibility and information content by the Sequencing Quality Control Consortium. Nat Biotechnol 2014, 32(9):903-914.

9. Baker M: 1,500 scientists lift the lid on reproducibility. Nature 2016, 533(7604):452454.

10. Mehta D: Highlight negative results to improve science. Nature 2019. 